\title{
Effect of supplementation with antihypertensive peptides from food proteins on cardiovascular risk factors: A systematic review and meta-analysis of randomised controlled trials
}

\author{
H.M. Cheng, A. Kazi, N. Mavroudis and J. Lara \\ Department of Applied Sciences, Faculty of Health and Life Sciences, University of Northumbria at Newcastle, \\ NE1 8ST, UK.
}

High blood pressure, particularly high systolic blood pressure, is the leading factors to global disability and disease ahead of smoking or high body mass index ${ }^{(1)}$. More effective nutritional and lifestyle-based interventions are thus required to prevent and manage high blood pressure.

In recent year, the health benefits of food proteins have attracted increased attention ${ }^{(2)}$. Bioactive peptides of animal and plant origin, for example derived from foods proteins such as milk and soybean, have antihypertensive properties by inhibiting ACE, which is responsible for the conversion of angiotensin I to angiotensin II. Earlier evidence on this topic has been previously reviewed, however the effect of such protein peptides on other cardiovascular risk factors has not been systematically reviewed. We undertook a systematic review and meta- analysis to evaluate the association between the peptides supplementation and blood pressure lowering effects. The protocol for this review has been registered with PROSPERO the International prospective register of systematic reviews (CRD42017080319).

A search of three major databases including Medline, Scopus and Web of science were thoroughly searched from inception till April 2018. A meta-analysis of intervention trials in adults ( $\geqslant$ than 18 years), reporting on the effect on cardiovascular risk factors including systolic blood pressure (SBP) and diastolic blood pressure (DBP) (measured under resting and ambulatory conditions), as well as blood lipids, inflammatory factors, and markers of endothelial function, was undertaken using random effects models.

Out of 1306 publications identified, 37 fulfilled inclusion criteria. 36 studies published between 1996 and 2016 including a total of 2586 individuals were meta-analysed. Peptide supplementation was associated with significant reduction of resting SBP (mean difference of $-2.71 \mathrm{mmHg}, 95 \%$ confidence interval [Cl] -3.90 to $-1.52, p<0.0001$; heterogeneity level assessed by the $I^{2}$ was $62 \%$ ) and DBP $(-1.35 \mathrm{mmHg}, \mathrm{CI}-2.04$ to $-0.65, p=0.006$, heterogeneity level was $36 \%)$. Subgroup analysis shows that peptide supplementation reduces resting SBP and DBP similarly in men and women, but effects are greater among hypertensive or individuals of Asian origin as opposed to normotensive or Caucasians. However, studies evaluating ambulatory blood pressure (ABP) are only showing non-statistically significant reductions. Evidence on the effect of peptides supplementation on total cholesterol, HDL-cholesterol-, LDL-cholesterol, VLDL-cholesterol, triglycerides, CRP, IL6, or PWV, indicated small but not statistically significant reductions.

In conclusion, this systematic review shows that there is evidence for a mild but significant antihypertensive effect of food protein peptides on resting SBP and DBP. However, current evidence from more recent studies measuring ABP are only showing nonstatistically significant reductions. In addition, current evidence on the effect of peptides supplementation on other cardiovascular risk factors is still scarce and suggests no effects. The antihypertensive effects of bioactive peptides require further evaluation in studies measuring ambulatory blood pressure, a more accurate marker of blood pressure, as well as on other markers of vascular risk, particularly endothelial function.

1. GBD (2016) Lancet 388, 1659-724.

2. Chanson-Rolle A, Aubin F, Braesco V et al. (2015) Plos One 10(11). 